\title{
Decomposition of Graphs into Paths and Cycles
}

\author{
S. Arumugam, ${ }^{1}$ I. Sahul Hamid, ${ }^{2}$ and V. M. Abraham ${ }^{3}$ \\ ${ }^{1}$ Core Group Research Facility (CGRF), National Center for Advanced Research in \\ Discrete Mathematics (n-CARDMATH), Kalasalingam University, Anand Nagar, Krishnankoil 626190, India \\ ${ }^{2}$ Department of Mathematics, The Madura College, Madurai 625 011, India \\ ${ }^{3}$ Department of Mathematics, Christ University, Bangalore 560 029, India
}

Correspondence should be addressed to V. M. Abraham; frabraham@christuniversity.in

Received 31 October 2012; Revised 12 March 2013; Accepted 12 March 2013

Academic Editor: Kinkar Ch Das

Copyright $\odot 2013$ S. Arumugam et al. This is an open access article distributed under the Creative Commons Attribution License, which permits unrestricted use, distribution, and reproduction in any medium, provided the original work is properly cited.

A decomposition of a graph $G$ is a collection $\psi$ of edge-disjoint subgraphs $H_{1}, H_{2}, \ldots, H_{r}$ of $G$ such that every edge of $G$ belongs to exactly one $H_{i}$. If each $H_{i}$ is a path or a cycle in $G$, then $\psi$ is called a path decomposition of $G$. If each $H_{i}$ is a path in $G$, then $\psi$ is called an acyclic path decomposition of $G$. The minimum cardinality of a path decomposition (acyclic path decomposition) of $G$ is called the path decomposition number (acyclic path decomposition number) of $G$ and is denoted by $\pi(G)\left(\pi_{a}(G)\right)$. In this paper we initiate a study of the parameter $\pi$ and determine the value of $\pi$ for some standard graphs. Further, we obtain some bounds for $\pi$ and characterize graphs attaining the bounds. We also prove that the difference between the parameters $\pi$ and $\pi_{a}$ can be made arbitrarily large.

\section{Introduction}

Graph decomposition problems rank among the most prominent areas of research in graph theory and combinatorics and further it has numerous applications in various fields such as networking, block designs, and bioinformatics.

A decomposition of a graph $G$ is a collection of edgedisjoint subgraphs $H_{1}, H_{2}, \ldots, H_{r}$ of $G$ such that every edge of $G$ belongs to exactly one $H_{i}$. Various types of path decompositions and corresponding parameters have been studied by several authors by imposing conditions on the paths in the decomposition. It is obvious that every graph admits a decomposition in which each subgraph $H_{i}$ is either a path or a cycle. In this connection, Erdös asked what is the minimum number of paths into which every connected graph on $n$ vertices can be decomposed and Gallai conjectured that this number is at most $\lceil n / 2\rceil$ as stated below.

Gallai's Conjecture (see [1]). If $G$ is a connected graph on $n$ vertices, then $G$ can be decomposed into $\lceil n / 2\rceil$ paths.

A good number of research articles have been published in which Gallai's is the focus of study and still this conjecture remains unsettled for more than 30 years. Towards a proof of the conjecture, Lovasz [1] made the first significant contribution by proving the following theorem.

Theorem 1 (see [1]). A graph $G$ on $n$ vertices (not necessarily connected) can be decomposed into $\lfloor n / 2\rfloor$ paths and cycles.

Gallai's conjecture and Theorem 1 motivate the following definition.

Definition 2. Let $\psi=\left\{H_{1}, H_{2}, \ldots, H_{r}\right\}$ be a decomposition of a graph $G$. If each $H_{i}$ is either a path or a cycle, then $\psi$ is called a path decomposition of $G$. If each $H_{i}$ is a path, then $\psi$ is called an acyclic path decomposition of $G$. The minimum cardinality of a path decomposition of $G$ is called the path decomposition number. Similarly the acyclic path decomposition number of $G$ is defined and is denoted by $\pi_{a}(G)$.

The parameter $\pi_{a}$ was introduced by Harary and further studied extensively by Harary and Schwenk [2], Péroche [3], Stanton et al. [4, 5], and Arumugam and Suseela [6]. This paper initiates a study of the parameter path decomposition number. 


\section{Basic Terminologies and Results}

By a graph $G=(V, E)$ we mean a finite, connected, and undirected graph without loops or multiple edges. For graph theoretic terminology we refer to Chartrand and Lesniak [7]. The order and size of a graph are denoted by $n$ and $m$, respectively.

If $P=\left(v_{1}, v_{2}, \ldots, v_{r}\right)$ is a path in a graph $G$, then the vertices $v_{2}, v_{3}, \ldots, v_{r-1}$ are called internal vertices of $P$ and $v_{1}$ and $v_{r}$ are called external vertices of $P$. For a cycle $C=$ $\left(v_{1}, v_{2}, \ldots, v_{r}, v_{1}\right)$, the vertex $v_{1}$ is called the initial vertex and all other vertices are internal vertices. Two paths $P$ and $Q$ of a graph $G$ are said to be internally disjoint if no vertex of $G$ is an internal vertex of both $P$ and $Q$. If $P=\left(v_{0}, v_{1}, v_{2}, \ldots, v_{r}\right)$ and $Q=\left(v_{r}=w_{0}, w_{1}, w_{2}, \ldots, w_{s}\right)$ are two paths in $G$, then the walk obtained by concatenating $P$ and $Q$ at $v_{r}$ is denoted by $P \circ Q$, that is, $P \circ Q=\left(v_{0}, v_{1}, \ldots, v_{r}=w_{0}, w_{1}, \ldots, w_{s}\right)$. Further when $P=\left(v_{1}, v_{2}, \ldots, v_{n}\right)$, the path $\left(v_{n}, v_{n-1}, \ldots, v_{1}\right)$ is denoted by $P^{-1}$.

A caterpillar is a tree in which removal of all the pendant vertices results in a path. For vertices $x$ and $y$ in a connected graph $G$, the detour distance $D(x, y)$ is the length of a longest $x-y$ path in $G$. The detour diameter $D$ of $G$ is defined to be $D=\max \{D(x, y): x, y \in V(G)\}$. Two graphs are said to be homeomorphic if both can be obtained from the same graph by a sequence of subdivisions of edges. The length of a longest cycle in a graph $G$ is called the circumference of $G$.

We need the following theorems.

Theorem 3 (see [4]). For any tree $T, \pi_{a}(T)=k / 2$, where $k$ is the number of vertices of odd degree.

Theorem 4 (see [6]). Let $G$ be a unicyclic graph with cycle $C$. Let $r$ denote the number of vertices of degree greater than two on $C$. Let $k$ denote the number of vertices of odd degree. Then

$$
\pi_{a}(G)= \begin{cases}\frac{2}{2}+1 & \text { if } r=0 \\ \frac{k}{2} & \text { otherwise }\end{cases}
$$

Theorem 5 (see [2]). For the complete graph $K_{n}$, one has $\pi_{a}\left(K_{n}\right)=\lceil n / 2\rceil$.

Theorem 6 (see [2]). For the complete bipartite graph $K_{r, s}$ with $r \leq s$, one has

$$
\pi_{a}\left(K_{r, s}\right)=\left\{\begin{array}{cl}
\frac{r+s}{2} & \text { if } r s \text { is odd } \\
\left\lceil\frac{r s}{2 r-\delta(r, s)}\right\rceil & \text { otherwise, }
\end{array}\right.
$$

where $\delta(r, s)$ is the conventional Kronecker delta.

\section{Bounds for $\pi$}

In this section we obtain some bounds for the path decomposition number $\pi$ and characterize the graphs attaining some of the bounds obtained. Let us first establish an upper and lower bound involving the order of the graph and the number of vertices of odd degree. For this we define the term $t(G)$ of a graph $G$. Given a path decomposition $\psi$ for a graph $G$, let $t_{\psi}=\sum_{P \in \psi} t(P)$, where $t(P)$ denotes the number of internal vertices of $P$. Now, $\operatorname{define} t(G)=\max t_{\psi}$, where the maximum is taken over all path decompositions $\psi$ of $G$.

Theorem 7. Let $G$ be a graph on $n$ vertices. Then $k / 2 \leq \pi(G) \leq$ $n / 2$, where $k$ is the number of vertices of odd degree in $G$.

Proof. Let $\mathscr{P}$ be the collection of all path decompositions of $G$ and let $\psi \in \mathscr{P}$.

Then

$$
\begin{aligned}
m & =\sum_{P \in \psi}|E(P)| \\
& =\sum_{P \in \psi}(t(P)+1) \\
& =\sum_{P \in \psi} t(P)+|\psi| \\
& =t_{\psi}+|\psi| .
\end{aligned}
$$

Hence, $|\psi|=m-t_{\psi}$ so that $\pi=m-t$. Also, it is not difficult to see that $m=k / 2+\sum_{v \in V(G)}\lfloor\operatorname{deg} v / 2\rfloor$ and hence $\pi(G)=$ $k / 2+\sum_{v \in V(G)}\lfloor\operatorname{deg} v / 2\rfloor-t$, which implies that $\pi(G) \geq k / 2$. The upper bound follows immediately from Theorem 1 .

Remark 8. If $G$ is a graph with $k$ vertices of odd degree, then $\pi(G)=k / 2$ if and only if there exists a path decomposition $\psi$ of $G$ such that every vertex $v$ is an internal vertex of $\lfloor\operatorname{deg} v / 2\rfloor$ members in $\psi$.

The following examples show that there are infinite families of graphs attaining the bounds given in the above theorem and consequently prove that these bounds are sharp. That is, the family of all trees and an infinite class of unicyclic graphs achieve the lower bound, whereas some common classes of graphs such as complete graphs, wheels, and complete bipartite graphs of odd size attain the upper bound. In this connection we need to note down the inequality that $\pi(G) \leq$ $\pi_{a}(G)$ which follows from the fact that every acyclic path decomposition of a graph is also a path decomposition.

Example 9. (i) For any tree $T, \pi(T)=\pi_{a}(T)=k / 2$.

(ii) If $G$ is a unicyclic graph with cycle $C$ and if $r$ denotes the number of vertices on $C$ with degree greater than two, then

$$
\pi(G)= \begin{cases}1 & \text { if } r=0 \\ \frac{k}{2}+1 & \text { if } r=1 \\ \frac{k}{2} & \text { otherwise. }\end{cases}
$$

Proof. (i) Since every path decomposition of $T$ is an acyclic path decomposition of $T$, we have $\pi(T)=\pi_{a}(T)$ and hence the result follows from Theorem 3 . 
(ii) If $r=0$, then $G=C$ so that $\pi(G)=1$. If $r=1$, then it follows from Theorem 4 that $\pi_{a}(G)=(k / 2)+1$ and hence $\pi(G) \leq \pi_{a}(G)=k / 2+1$. Further, for any path decomposition $\psi$ of $G$ all the $k$ odd vertices and at least one vertex on $C$ are external vertices of some paths in $\psi$ so that $\pi(G) \geq k / 2+1$. Thus $\pi(G)=k / 2+1$. If $r>1$, by Theorem 4 , we have $\pi_{a}(G)=$ $k / 2$ and hence $\pi(G) \leq \pi_{a}(G)=k / 2$. Thus $\pi(G)=k / 2$ as $\pi(G) \geq k / 2$.

Example 10. (i) For the complete graph $K_{n}$, we have $\pi\left(K_{n}\right)=$ $\lfloor n / 2\rfloor$.

(ii) For the complete bipartite graph $K_{r, s}$ with $r \leq s$

$$
\pi\left(K_{r, s}\right)= \begin{cases}\frac{r+s}{2} & \text { if } r s \text { is odd, } \\ \left\lceil\frac{s}{2}\right] & \text { otherwise, }\end{cases}
$$

(iii) If $W_{n}$ denotes the wheel on $n$ vertices, then $\pi\left(W_{n}\right)=$ $\lfloor n / 2\rfloor$.

Proof. (i) Let $\psi$ be any path decomposition of $K_{n}$. Since every member of $\psi$ covers at most $n$ edges, we have $|\psi| \geq\lfloor n / 2\rfloor$ so that $\pi\left(K_{n}\right) \geq\lfloor n / 2\rfloor$. Further, it follows from Theorem 1 that $\pi\left(K_{n}\right) \leq\lfloor n / 2\rfloor$. Thus $\pi\left(K_{n}\right)=\lfloor n / 2\rfloor$.

(ii) Assume first that $r=s$. Now, if $r$ is odd, then every vertex is of odd degree and hence $\pi\left(K_{r, r}\right)=r$. If $r$ is even, then $K_{r, r}$ can be decomposed into hamiltonian cycles and hence $\pi\left(K_{r, r}\right) \leq r / 2$. Further, for any path decomposition $\psi$ of $K_{r, r}$, every member of $\psi$ covers at most $2 r$ edges so that $|\psi| \geq r^{2} / 2 r=r / 2$ and hence $\pi\left(K_{r, r}\right) \geq r / 2$. Thus $\pi\left(K_{r, r}\right)=r / 2$.

Suppose $r \neq s$. If $r s$ is odd, then every vertex is of odd degree and hence $\pi\left(K_{r, s}\right)=(r+s) / 2$. If $r s$ is even, then $\pi\left(K_{r, s}\right) \leq \pi_{a}\left(K_{r, s}\right)=\lceil r s / 2 r\rceil=\lceil s / 2\rceil$. Further, for any path decomposition $\psi$ of $K_{r, s}$, every member in $\psi$ covers at most $2 r$ edges and hence $|\psi| \geq\lceil r s / 2 r\rceil=\lceil s / 2\rceil$. Thus $\pi\left(K_{r, s}\right)=\lceil s / 2\rceil$.

(iii) Since the number of vertices of odd degree in $W_{n}$ is $n-1$ or $n$ accordingly as $n$ is odd or even, it follows from Theorem 7 that $\pi\left(W_{n}\right) \geq\lfloor n / 2\rfloor$. Also by Theorem 1 , we have $\pi\left(W_{n}\right) \leq\lfloor n / 2\rfloor$ and hence $\pi\left(W_{n}\right)=\lfloor n / 2\rfloor$.

We now proceed to obtain some lower and upper bounds for $\pi$ involving detour diameter, circumference, and maximum degree of a graph along with the characterization of graphs attaining the bounds.

Theorem 11. For any graph $G, \pi(G) \leq m-D+1$, where $D$ is the detour diameter of $G$. Further, equality holds if and only if $G$ is a caterpillar with $\Delta \leq 3$.

Proof. Let $P$ be a path in $G$ of length $D$. Then $\psi=\{P\} \cup\{E(G)-$ $E(P)\}$ is a path decomposition of $G$ and $|\psi|=m-D+1$. Hence, $\pi(G) \leq m-D+1$

Suppose $\pi(G)=m-D+1$. Let $P$ be a path of length $D$ in $G$. If there is a vertex $v$ not on $P$ with $\operatorname{deg} v \geq 2$, let $Q$ be a longest path which contains $v$ and edge-disjoint from $P$. Thus, $\psi=\{P, Q\} \cup S$, where $S$ is the set of edges not covered by the paths $P$ and $Q$ is a path decomposition of $G$ and $|\psi|<$
$m-D+1$, which is a contradiction. Thus every vertex not on $P$ is a pendent vertex and hence $G$ is a caterpillar.

Now, if there exists a vertex $w$ on $P$ with $\operatorname{deg} w \geq 4$, let $w_{1}$ and $w_{2}$ be two vertices not on $P$ which are adjacent to $w$. Then $\psi_{1}=\left\{P, P_{1}=\left(w_{1}, w, w_{2}\right)\right\} \cup S$, where $S$ is the set of edges not covered by the paths $P$ and $P_{1}$ is a path decomposition of $G$ and $|\psi|<m-D+1$, which is a contradiction. Hence, $\operatorname{deg} w \leq 3$, for all $w \in V(P)$.

The converse is obvious.

Theorem 12. For any graph $G, \pi(G) \leq m-c+1$, where $c$ is the circumference of $G$. Further, equality holds if and only if $G$ is either a cycle or a cycle with exactly one chord or a unicyclic graph with $\Delta=3$ and every vertex not on the cycle is a pendant vertex.

Proof. Let $C$ be a cycle of length $c$. Then $\psi=\{C\} \cup\{E(G)-$ $E(C)\}$ is a path decomposition of $G$ and $|\psi|=m-c+1$. Hence $\pi(G) \leq m-c+1$.

Suppose $\pi=m-c+1$. Let $C$ be a cycle of length $c$ in $G$. We consider the following two cases.

Case $1(V(G)=V(C))$. We claim that $C$ has at most one chord. Suppose $C$ has two chords say $e_{1}$ and $e_{2}$. If $e_{1}=v w_{1}$ and $e_{2}=v w_{2}$, then $\psi=\left\{C,\left(w_{1}, v, w_{2}\right)\right\} \cup S$, where $S$ is the set of edges not covered by $C$ and the path $\left(w_{1}, v, w_{2}\right)$ is a path decomposition of $G$ and $|\psi|<m-c+1$, which is a contradiction. Suppose $e_{1}=v_{1} w_{1}$ and $e_{2}=v_{2} w_{2}$. Let $P$ and $Q$ denote the two $\left(v_{1}, v_{2}\right)$-sections of the cycle $C$ and without loss of generality we assume that $P$ contains one of the $\left(w_{1}, w_{2}\right)$-sections of $C$. Now, let $Q_{1}$ denote the path consisting of the edge $w_{1} v_{1}$, followed by the path $Q$ and the edge $v_{2} w_{2}$. Then $\psi_{1}=\left\{P, Q_{1}\right\} \cup S$, where $S$ denotes the set of edges not covered by the paths $P$ and $Q_{1}$ is a path decomposition of $G$ and $\left|\psi_{1}\right|<m-c+1$, which is a contradiction. Hence, $C$ has at most one chord. Thus $G$ is a cycle or a cycle with exactly one chord.

Case $2(V(G) \neq V(C))$. Suppose that there exists a vertex $v$ not on $C$ with $\operatorname{deg} v \geq 2$. Let $Q$ be a longest path which contains $v$ and edge-disjoint from $C$. Thus $\psi=\{C, Q\} \cup S$, where $S$ is the set of edges not covered by $C$ and $Q$ is a path decomposition of $G$ and $|\psi|<m-c+1$, which is a contradiction. Thus every vertex not on $C$ is a pendant vertex.

If there exists a vertex $v$ on $C$ with $\operatorname{deg} v \geq 4$, let $P$ be a longest path containing $v$ and edge-disjoint from $C$. Then $\psi=\{C, P\} \cup S$, where $S$ is the set of edges not covered by $C$ and $P$ is a path decomposition of $G$ with $|\psi|<m-c+1$, which is a contradiction. Hence every vertex on $C$ has degree less than or equal to 3 .

Now, we claim that the cycle $C$ has no chord. Suppose $v w$ is a chord of $C$. Let $y$ be a pendant vertex not on $C$. Then there exists a vertex $x$ on $C$ different from the vertices $v$ and $w$ such that $x$ and $y$ are adjacent. Let $P$ and $Q$ denote the two $(x, w)$-sections of $C$ and without loss of generality we assume that $v$ lies on $P$. Let $Q_{1}$ be the path consisting of the edge $y x$ followed by the path $Q$ and the edge $w v$. Now, $\psi=\left\{P, Q_{1}\right\} \cup S$, where $S$ is the set of edges not covered by the paths $P$ and $Q_{1}$ 
is a path decomposition of $G$ and $|\psi|<m-c+1$, which is a contradiction. Hence, $C$ has no chords.

Thus $G$ is a unicyclic graph with $\Delta=3$ and every vertex not on the cycle is a pendant vertex. The converse is obvious.

Remark 13. Obviously, for any vertex $v$ of a graph $G$, at most two of the edges incident with $v$ can be covered by a member of a path decomposition of $G$. As this is true in particular for a vertex of maximum degree it follows that $\pi(G) \geq\lceil\Delta / 2\rceil$. This bound is sharp as a complete graph of odd order attains this. In fact, every graph which is hamiltonian cycle decomposable attains the bound. Further, for any tree $T, \pi(T)=\lceil\Delta / 2\rceil$ if and only if $T$ is homeomorphic to the star $K_{1, \Delta}$.

The following theorem characterizes the family of unicyclic graphs achieving the bound given in Remark 13.

Theorem 14. Let $G$ be a unicyclic graph with cycle $C$ and $G \neq C$. Then $\pi(G)=\lceil\Delta / 2\rceil$ if and only if every vertex not on $C$ has degree 1 or 2 , at most two vertices on $C$ have degree $\geq 3$ and $C$ has exactly one vertex with degree $\geq 3$ if $\Delta \geq 5$.

Proof. Let $G$ be a unicyclic graph with $\pi(G)=\lceil\Delta / 2\rceil$. Let $\psi$ be a minimum path decomposition of $G$. Let $v$ be a vertex in $G$ with $\operatorname{deg} v=\Delta$. Then every member of $\psi$ passes through $v$.

We claim that $v$ lies on the cycle $C$. This is obvious if $C \epsilon$ $\psi$. If $C \notin \psi$, let $P_{1}$ and $P_{2}$ be the paths in $\psi$ covering the edges of the cycle $C$. Since both $P_{1}$ and $P_{2}$ pass through $v$; it follows that $v$ lies on $C$. Thus every vertex of degree $\Delta$ lies on $C$ and consequently every vertex not on $C$ has degree 1 or 2 .

Further if $\Delta(G) \geq 5$, it follows that $G$ contains exactly one vertex of degree $\Delta$ and if $\Delta(G) \leq 4$, then $G$ contains at most two vertices of degree $\geq 3$.

The converse is obvious.

In the following theorem we characterize graphs of order $n$ with $\pi=\lceil\Delta / 2\rceil$ and having minimum (maximum) number of edges.

Theorem 15. Let $G$ be a graph of order $n$ with $\pi(G)=\lceil\Delta / 2\rceil$. Then $n-1 \leq m \leq\lceil n \Delta / 2\rceil$. Further, $m=n-1$ if and only if $G$ is homeomorphic to the star $K_{1, \Delta}$. Also $m=\lceil n \Delta / 2\rceil$ if and only if $G$ is hamiltonian cycle decomposable or $G$ is the complete graph $K_{n}$ with $n$ even.

Proof. Let $G$ be a graph with $\pi(G)=\lceil\Delta / 2\rceil$. Then $G$ is connected and hence $n-1 \leq m \leq\lceil n \Delta / 2\rceil$.

Now, suppose $m=n-1$. Then $G$ is a tree. As $\pi(G)=$ $\lceil\Delta / 2\rceil$ it follows that $G$ is homeomorphic to the star $K_{1, \Delta}$. Conversely, if $G$ is homeomorphic to $K_{1, \Delta}$, then $\pi(G)=$ $\lceil\Delta / 2\rceil$.

Now, suppose $m=\lceil n \Delta / 2\rceil$. Then $G$ is $\Delta$-regular. If $\Delta$ is even, then for any minimum path decomposition $\psi$ of $G$, we have $|\psi|=\Delta / 2$ and each path in $\psi$ must cover $n$ edges so that $G$ is hamiltonian cycle decomposable. Conversely, if $G$ is hamiltonian cycle decomposable, then $\pi(G)=\Delta / 2$. If $\Delta$ is odd, then $\pi(G)=n / 2=\lceil\Delta / 2\rceil$ and hence $\Delta=n-1$. Hence, $G=K_{n}$ and $n$ is even. The converse follows from Example 10(i).

\section{Relation between the Parameters $\pi$ and $\pi_{a}$}

In this section we establish some relation between the parameters $\pi$ and $\pi_{a}$.

Theorem 16. For any graph $G$, one has $\pi(G) \leq \pi_{a}(G) \leq$ $2 \pi(G)$. Further, $\pi_{a}(G)=2 \pi(G)$ if and only if every component of $G$ is a cycle.

Proof. The first inequality is already seen. For the other inequality, consider a path decomposition $\psi$ of $G$ and spilt each cycle member of $\psi$ into two paths and thus an acyclic path decomposition of $G$ with cardinality at most $2 \pi(G)$ is obtained, which proves the required inequality. Now suppose $\pi_{a}(G)=2 \pi(G)$. Let $\psi$ be a minimum path decomposition of $G$. Then every member of $\psi$ is a cycle. Further, all the cycles in $\psi$ are vertex-disjoint and hence every component of $G$ is a cycle. The converse is obvious.

Remark 17. The first part of the inequalities given in the above theorem is strict in the sense that they both are equal for infinitely many graphs. For example, one has the following.

(i) For even integers $n$, we have $\pi\left(K_{n}\right)=\pi_{a}\left(K_{n}\right)$, which follows from Theorem 5 and Example 10(i).

(ii) Example 9 and Theorem 4 together show that the parameters $\pi$ and $\pi_{a}$ are equal for all trees and also for the class of all unicyclic graphs other than the cycles.

Though the upper bound suggested by Gallai's conjecture for $\pi_{a}$ and the upper bound given by Theorem 1 for $\pi$ differ by at most 1 , the difference between the actual values of these parameters can be made arbitrarily large, which we proceed to prove. The following lemma is useful in this regard.

Lemma 18. Let $G$ be a connected graph which can be decomposed into a cycle $C=\left(v_{1}, v_{2}, \ldots, v_{r}, v_{1}\right)$ and a path $P=$ $\left(w_{1}, \ldots, w_{s}\right)$. Then $G$ can be decomposed into two paths if and only if $G$ has a vertex of degree 2 which lies on $C$ or on the $\left(w_{i_{1}}, w_{i_{2}}\right)$-section of $P$ where $i_{1}$ is the smallest positive integer such that $1 \leq i_{1}<s$ and $w_{i_{1}} \in V(C)$ and $i_{2}$ is the largest positive integer such that $i_{1}<i_{2} \leq s$ and $w_{i_{2}} \in V(C)$.

Proof. Suppose $G$ can be decomposed into two paths $P_{1}$ and $P_{2}$. Since $w_{1}$ and $w_{s}$ are the only two vertices of odd degree in $G$, we may assume that $w_{1}$ is the origin of $P_{1}$ and $w_{s}$ is the origin of $P_{2}$. Let $v$ be the terminus of $P_{1}$. Since the degree of $v$ is even, it follows that $v$ is not an internal vertex of $P_{2}$ so that $v$ is the terminus of $P_{2}$ as well, and the degree of $v$ is 2. Clearly $v$ cannot lie on the $\left(w_{1}, w_{i_{1}}\right)$-section or the $\left(w_{i_{2}}, w_{s}\right)$-section of $P$.

Conversely, suppose $G$ has a vertex $v$ of degree two satisfying the conditions stated in the lemma. We consider two cases.

Case $1(v \in V(C))$. Since $G$ is connected, $|V(P) \cap V(C)| \geq 1$. If $|V(P) \cap V(C)|=1$, then trivially $G$ can be decomposed into two paths. Hence, we assume that $|V(P) \cap V(C)| \geq 2$. If there exists a $\left(v, w_{i_{1}}\right)$-section of $C$ which is internally disjoint from $P$, let $P_{1}$ be the path consisting of the $\left(w_{1}, w_{i_{1}}\right)$-section of 
$P$ followed by the $\left(w_{i_{1}}, v\right)$-section of $C$ which is not internally disjoint from $P$ and let $P_{2}$ be the path consisting of the $\left(\nu, w_{i_{1}}\right)$ section of $C$ which is internally disjoint from $P$ followed by the $\left(w_{i}, w_{s}\right)$-section of $P$. Then $\left\{P_{1}, P_{2}\right\}$ is a decomposition of $G$. The proof is similar if there exists a $\left(v, w_{i_{2}}\right)$-section of $C$ which is internally disjoint from $P$.

Now, suppose both the $\left(v, w_{i_{1}}\right)$-sections of $C$ and both the $\left(v, w_{i_{2}}\right)$-sections of $C$ are not internally disjoint from $P$. Let $r$ be the largest positive integer such that $i_{1}<r<i_{2}$ and $w_{r} \in$ $V(C)$. Then the $\left(w_{r}, w_{i_{2}}\right)$-section of $P$ and one of the $\left(w_{r}, w_{i_{2}}\right)$ sections of $C$, say $Q$, are internally disjoint. If $Q$ has length greater than 1 , then any internal vertex $v_{i}$ of $Q$ has degree 2 and the $\left(v_{i}, w_{i_{2}}\right)$-section of $Q$ is internally disjoint from $P$ and hence the proof is complete. If the $\left(w_{r}, w_{i_{2}}\right)$-section of $P$ has length greater than 1 , let $P_{1}$ be the path consisting of the $\left(w_{s}, w_{i_{2}}\right)$-section of $P^{-1}$ followed by the $\left(w_{i_{2}}, w_{r}\right)$-section of $C$ which is different from $Q$ followed by the edge $w_{r} w_{r+1}$ and let $P_{2}$ be the path consisting of the $\left(w_{r+1}, w_{i_{2}}\right)$-section of $P$ followed by $Q^{-1}$ followed by $\left(w_{r}, w_{1}\right)$-section of $P^{-1}$. Then $\left\{P_{1}, P_{2}\right\}$ is a decomposition of $G$.

Case 2 (no vertex on $C$ has degree 2). In this case $v$ lies on the $\left(w_{i_{1}}, w_{i_{2}}\right)$-section of $P$. Let $v=w_{i}$ and let $a$ and $b$ be integers with $i_{1} \leq a<i<b \leq i_{2}$ such that $w_{a}, w_{b} \in V(C)$ and no internal vertex of the $\left(w_{a}, w_{b}\right)$-section of $P$ lies on $C$. Without loss of generality we assume that there exists a $\left(w_{i_{1}}, w_{b}\right)$-section of $C$, say $Q_{1}$, which contains $w_{a}$ and $w_{i_{2}}$ and let $Q_{2}$ be the other $\left(w_{i_{1}}, w_{b}\right)$-section of $C$. Let $P_{1}$ be the path consisting of the $\left(w_{1}, w_{i_{1}}\right)$-section of $P$, followed by $Q_{1}$ and the $\left(w_{b}, w_{i}\right)$-section of $P^{-1}$. Let $P_{2}$ be the path consisting of the $\left(w_{s}, w_{b}\right)$-section of $P^{-1}$ followed by $Q_{2}^{-1}$ and the $\left(w_{i_{2}}, w_{i}\right)$ section of $P$. Then $\left\{P_{1}, P_{2}\right\}$ is a decomposition of $G$.

Corollary 19. If $G$ is a graph admitting a minimum path decomposition $\psi$ having exactly one cycle $C=\left(v_{1}, \ldots, v_{r}, v_{1}\right)$, then $\pi_{a} \leq \pi+1$. Further, $\pi_{a}=\pi$ if and only if there exists a path $P=\left(w_{1}, \ldots, w_{s}\right)$ in $\psi$ with $V(P) \cap V(C) \neq \emptyset$ and the induced subgraph $\langle V(C) \cup V(P)\rangle$ satisfies the conditions stated in Lemma 18.

Theorem 20. Given any positive integer $k$, there exists a graph $G$ such that $\pi_{a}(G)-\pi(G)=k$.

Proof. Let $G$ be the graph consisting of $k$ vertex-disjoint cycles $C_{1}, C_{2}, \ldots, C_{k}$ and a $u-v$ path $P=\left(u, v_{11}, \ldots, v_{1 n_{1}}\right.$, $\left.v_{21}, \ldots, v_{2 n_{2}}, \ldots, v_{k_{1}}, v_{k_{2}}, \ldots, v_{k n_{k}}, v\right)$, where $V\left(C_{j}\right)=\left\{v_{j_{1}}\right.$, $\left.v_{j_{2}}, \ldots, v_{j n_{j}}\right\}$, for $j=1,2, \ldots, k$. Clearly $\pi(G)=k+1$. Further, $\pi_{a}(G) \leq 2 k+1$. We now prove that $\pi_{a}(G) \geq 2 k+1$ by induction on $k$.

If $k=1$, then it follows from Lemma 18 that $\pi_{a}(G)=$ $3=2 k+1$. Assume that $\pi_{a}(G) \geq 2 r+1$, for all graphs $G$ as described above with $r<k$ cycles. Let $G$ be such a graph with $k$ cycles. Let $\psi$ be an acyclic path decomposition of $G$. Let $P_{1}$ be the path in $\psi$ containing the edge $v_{1 n_{1}} v_{21}$. Let $G_{1}=$ $\left\langle V\left(C_{1}\right) \cup\left\{u, v_{21}\right\}\right\rangle, G_{2}=\left\langle V\left(C_{2}\right) \cup V\left(C_{3}\right) \cup \cdots \cup V\left(C_{n}\right) \cup\left\{v, v_{1 n_{1}}\right\}\right\rangle$, $H_{1}=G_{1}-E\left(P_{1}\right)$ and $H_{2}=G_{2}-E\left(P_{1}\right)$. Let $P_{1}^{\prime}, P_{2}^{\prime}, \ldots, P_{r}^{\prime}$ be the paths in $\psi$ which decompose the graph $H_{1}$ and let $P_{1}^{\prime \prime}, P_{2}^{\prime \prime}, \ldots, P_{s}^{\prime \prime}$ be the paths in $\psi$ which decompose the graph
$H_{2}$. Clearly $r \geq 2$. We now claim that $s \geq 2 k-2$. Let $w \neq v_{1 n_{1}}$ be the end vertex of $P_{1}$ which lies in $G_{2}$. Then the $\left(v_{1 n_{1}}, w\right)$-section of $P_{1}$ together with the paths $P_{1}^{\prime \prime}, P_{2}^{\prime \prime}, \ldots, P_{s}^{\prime \prime}$ decompose the graph $G_{2}$. Hence, by induction hypothesis $s+1 \geq \pi_{a}\left(G_{2}\right) \geq 2(k-1)+1=2 k-1$ so that $s \geq 2 k-2$. Hence, $|\psi|=r+s+1 \geq 2+2 k-2+1=2 k+1$ so that $\pi_{a}(G) \geq 2 k+1$. Thus $\pi_{a}(G)=2 k+1$ and so $\pi_{a}(G)-\pi(G)=k$.

Theorem 21. Let $G$ be a connected graph. Then $\pi_{a}(G)=$ $2 \pi(G)-1$ if and only if $G$ is the graph consisting of $\pi-$ 1 vertex-disjoint cycles $C_{1}, C_{2}, \ldots, C_{\pi-1}$ and a path $P=$ $\left(w_{1}, w_{2}, \ldots, w_{r}\right)$ satisfying the following conditions.

(i) Every vertex of the cycle $C_{i}, 1 \leq i \leq \pi-1$, lies on the path $P$.

(ii) If $i_{1}$ and $i_{2}$ denote, respectively, the smallest and the largest positive integer such that $w_{i_{1}}, w_{i_{2}} \in V\left(C_{i}\right), 1 \leq$ $i \leq \pi-1$, then every edge of the $\left(w_{i_{1}}, w_{i_{2}}\right)$-section of $P$ is a chord of the cycle $C_{i}$.

Proof. Suppose $\pi_{a}(G)=2 \pi(G)-1$. Let $\psi$ be a minimum path decomposition of $G$. Then $\psi$ consists of exactly one path, say $P$ and $\pi(G)-1$ vertex-disjoint cycles, say $C_{1}, C_{2}, \ldots, C_{\pi(G)-1}$. Let $P=\left(w_{1}, w_{2}, \ldots, w_{r}\right)$. Since $G$ is connected, $V(P) \cap V\left(C_{i}\right) \neq \emptyset$ for all $i, 1 \leq i \leq \pi(G)-1$. Now, if there exists a vertex of degree 2 in $G$ which lies either on any of the cycles $C_{i}$ or on the $\left(w_{i_{1}}, w_{i_{2}}\right)$-section of $P$ then it follows from Lemma 18 that $\pi_{a}(G) \leq 2 \pi(G)-2$, which is a contradiction. Hence, (i) and (ii) hold.

Now, the graph $G$ given in the theorem is nothing but the graph described in Corollary 19 with $k=\pi-1$ and thus the converse follows.

Theorem 22. Given two positive integers $a$ and $b$ with $a \leq b \leq$ $2 a$, there exists a graph $G$ such that $\pi(G)=a$ and $\pi_{a}(G)=b$.

Proof. If $b=2 a$, let $G$ be the graph with $a$ components, each component being a cycle. If $b=2 a-1$, let $G$ be the graph given in Theorem 21. If $a=b$, let $G$ be the graph consisting of exactly one cut vertex and $a$ blocks, each block being a cycle. In all these cases, $\pi(G)=a$ and $\pi_{a}(G)=b$.

Now, suppose $a+1 \leq b \leq 2 a-1$. Let $b=a+r$, where $1 \leq r \leq a-1$. Let $G$ be the connected graph consisting of $a-1$ vertex-disjoint cycles $C_{1}, C_{2}, \ldots, C_{a-1}$ and a path $P=$ $\left(w_{1}, w_{2}, \ldots, w_{s}\right)$ satisfying the following conditions:

(i) $V(P) \cap V\left(C_{i}\right)=V\left(C_{i}\right)$ if $1 \leq i \leq r$ and $V(P) \cap V\left(C_{i}\right)$ is a proper nonempty subset of $V\left(C_{i}\right)$ if $r+1 \leq i \leq b$;

(ii) if $i_{1}$ and $i_{2}$ denote, respectively, the smallest and largest positive integer such that $w_{i_{1}}, w_{i_{2}} \in V\left(C_{i}\right)$, $1 \leq i \leq r$, then each edge of the $\left(w_{i_{1}}, w_{i_{2}}\right)$-section of $P$ is a chord of the cycle $C_{i}$.

Clearly $\pi(G)=a$ and it follows from Lemma 18 that $\pi_{a}(G)=$ $2 r+1+a-1-r=r+a=b$.

We conclude the paper by posing the following problem for further researchs.

(i) Characterize graphs $G$ for which $\pi(G)=k / 2$, where $k$ is the number of vertices of odd degree in $G$. 
(ii) Characterize graphs $G$ for which $\pi(G)=\lfloor n / 2\rfloor$.

(iii) Characterize graphs $G$ for which $\pi_{a}(G)=\pi$.

(iv) Characterize graphs for which $\pi(G)=\lceil\Delta / 2\rceil$.

\section{References}

[1] L. Lovasz, "On covering of graphs," in Theory of Graphs, P. Erdös and G. Katona, Eds., Procedure Collage, pp. 231-236, Academic Press, Tihany, Hungary, 1968.

[2] F. Harary and A. J. Schwenk, "Evolution of the path number of a graph: covering and packing in graphs II," in Graph Theory and Computing, C. Road, Ed., pp. 39-45, Academic Press, New York, NY, USA, 1972.

[3] B. Péroche, "The path-numbers of some multipartite graphs," Annals of Discrete Mathematics, vol. 9, pp. 195-197, 1980.

[4] R. G. Stanton, D. D. Cowan, and L. O. James, "Some results on path numbers," in Proceedings of the Louisiana Conference on Combinatorics, Graph Theory and computing, pp. 112-1135, 1970.

[5] R. G. Stanton, L. O. James, and D. D. Cowan, "Tripartite path numbers," in Graph Theory and Computing, R. C. Read, Ed., pp. 285-294, Academic Press, New York, NY, USA, 1972.

[6] S. Arumugam and J. S. Suseela, "Acyclic graphoidal covers and path partitions in a graph," Discrete Mathematics, vol. 190, no. 1-3, pp. 67-77, 1998.

[7] G. Chartrand and L. Lesniak, Graphs \& Digraphs, CRC Press, Boca Raton, Fla, USA, 4th edition, 2005. 


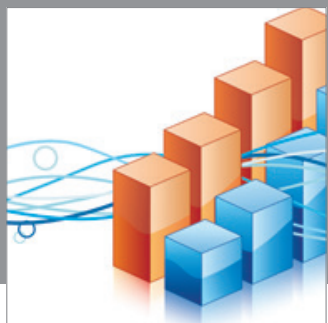

Advances in

Operations Research

mansans

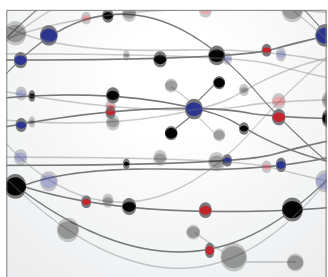

The Scientific World Journal
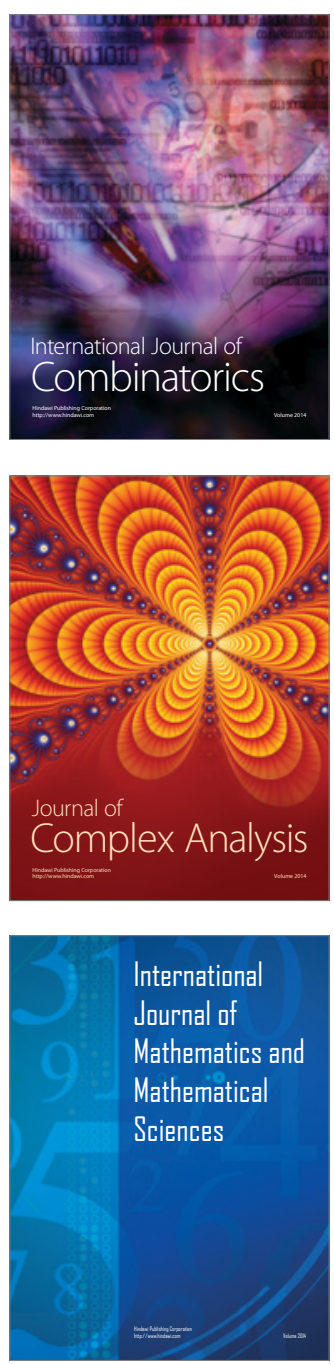
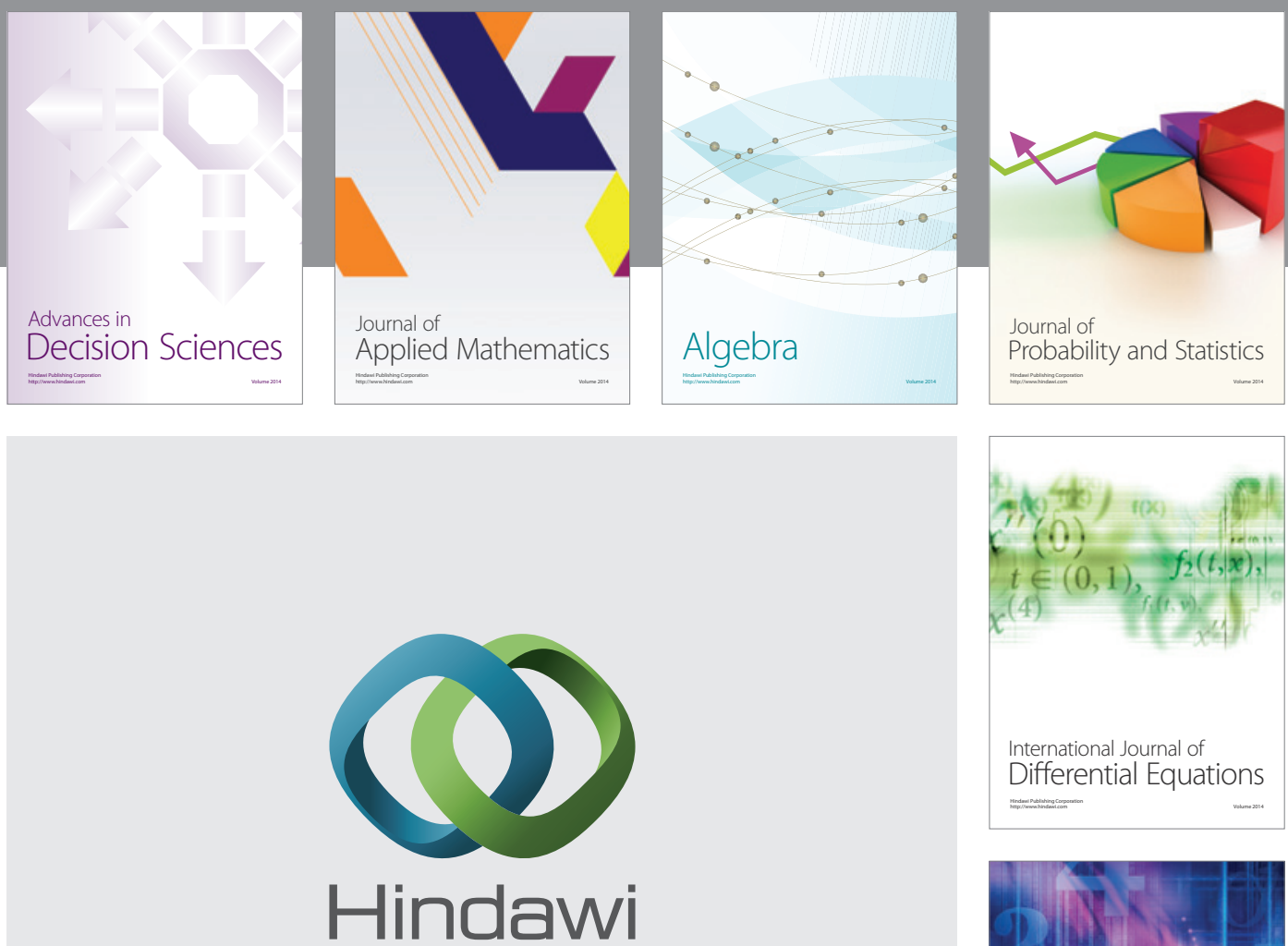

Submit your manuscripts at http://www.hindawi.com
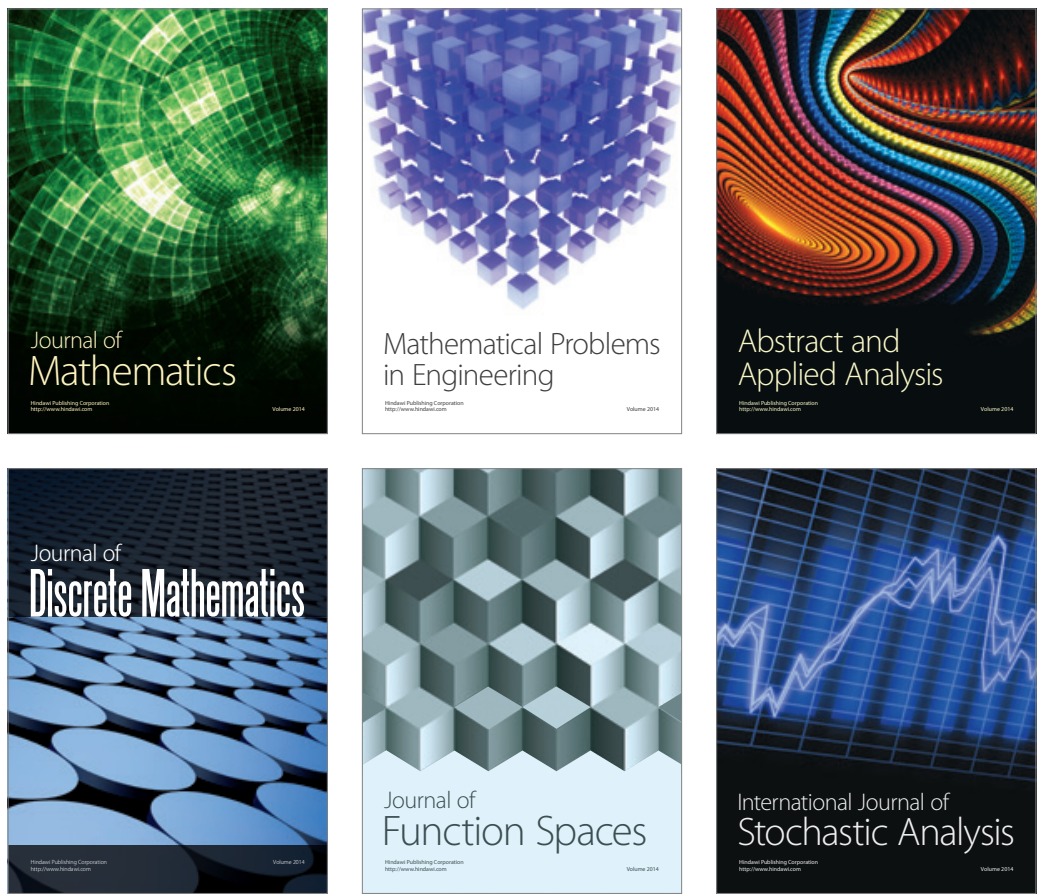

Journal of

Function Spaces

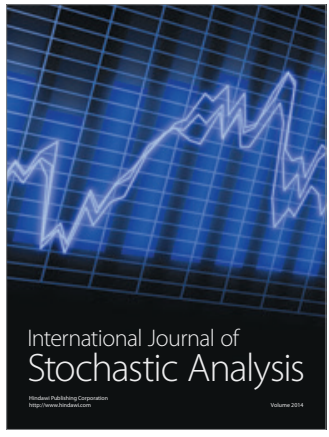

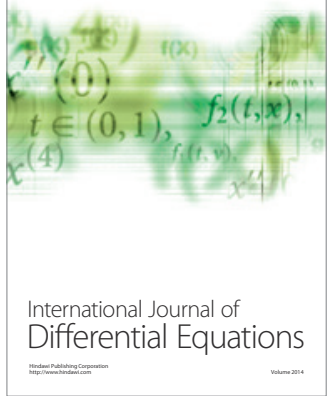
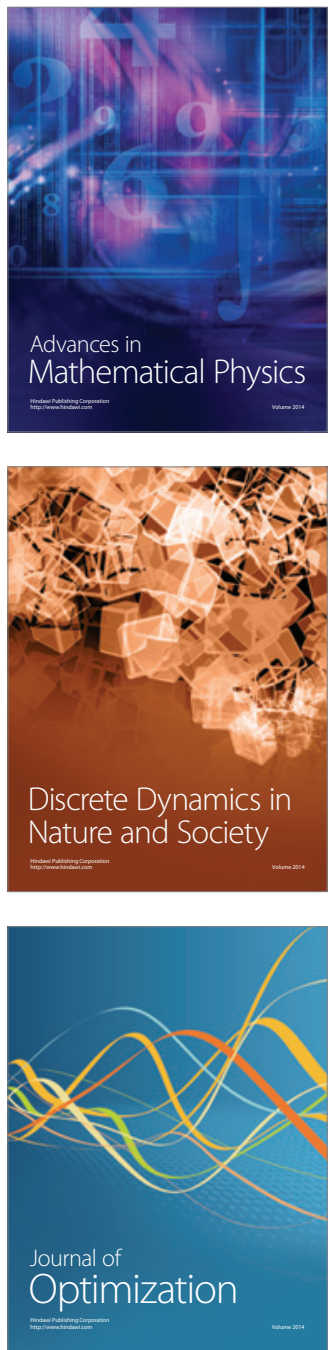